TRANSACTIONS OF THE

AMERICAN MATHEMATICAL SOCIETY

Volume 188, Issue 2, 1974

\title{
SOME COMBINATORIAL PRINCIPLES
}

BY

\section{JUSSI KETONEN}

\begin{abstract}
We extend some large cardinal axioms of Jensen to weakly inaccessible cardinals. Related problems regarding the saturatedness of certain filters are also studied.
\end{abstract}

1. Introduction and definitions. We shall generalize some notions considered in R. Jensen [1]. Our notation and terminology conforms with that used in the most recent set-theoretic literature. Thus, for example, small greek letters $\alpha, \beta, \ldots$ are used to denote ordinals and $|X|$ always denotes the cardinality of the set $X$. The letter $\kappa$ always denotes a cardinal number. A subset $X \subseteq \kappa$ is Mahlo if it intersects every closed unbounded subset of $\kappa$. Most of the results in this paper were obtained during the author's stay in S.U.N.Y at Buffalo. The author wishes to express his gratitude to William Boos and Jack Silver for many helpful discussions.

The following concepts are of fundamental importance in this paper.

1.1. Definition. A regular cardinal $\kappa$ is subtle if for every closed unbounded set $C \subseteq \kappa$, sequence $\left(S_{\alpha} \mid a<\kappa\right)$ of sets so that $S_{\alpha} \subseteq \alpha(a<\kappa)$, there exist elements $\alpha, \beta \in C$ so that $\alpha<\beta$ and $S_{\beta} \cap \alpha=S_{\alpha}$.

1.2. Definition. A regular cardinal $\kappa$ is ethereal if for every closed unbounded set $C \subseteq \kappa$, sequence $\left(S_{\alpha} \mid a<\kappa\right)$ of sets so that for $\alpha<\kappa,\left|S_{\alpha}\right|=|a|$ and $S_{\alpha} \subseteq \alpha$ there exist elements $\alpha, \beta \in C$ so that

$$
a<\beta \text { and }\left|S_{\alpha} \cap S_{\beta}\right|=|\alpha| \text {. }
$$

1.3. Definition. A filter $F$ on $\kappa$ is $\lambda$-saturated if there is no family $\left\{X_{a} \mid a<\lambda\right\}$ of subsets of $k$ so that

$$
\alpha<\beta<\lambda \rightarrow-X_{a} \notin F \text { and }-\left(X_{\alpha} \cap X_{\beta}\right) \dot{\epsilon} F \text {. }
$$
$f(a)<a$.

1.4. Definition. (1) A function $f$ on $\kappa$ is pressing down if, for every $\omega<\alpha<\kappa$,

(2) A filter $F$ on $\kappa$ is normal if every pressing down function on a set of positive $F$-measure is a constant on a set of positive $F$-measure.

Received by the editors December 20, 1972.

AMS (.MOS) subject classifications (1970). Primary 02K35; Secondary 02K05.

Key words and phrases. Mahlo, saturated filters, normal filters.

Copyright O 1974. American Mathematical Society 
1.5. Definition. For a regular $\kappa, \diamond(\kappa)$ denotes the following statement:

There exist sets $\left(S_{a} \mid \alpha \subset \kappa\right), S_{\alpha} \subseteq \alpha(\alpha<\kappa)$ so that for any $X \subseteq \kappa$ the set $\left\{a \mid X \cap \alpha=S_{\alpha}\right\}$ is Mahlo.

Definitions 1.1 and 1.5 are due.to $R$. Jensen (see [1]). For more on saturated and normal filters, see Solovay [3]. It is easy to see that Definition 1.2 is a natural generalization of Definition 1.1; every subtle cardinal is obviously ethereal and if $\kappa$ is ethereal, $\kappa$ is weakly inaccessible: For if $\tau$ is a cardinal so that $\kappa=\tau^{+}$, we can find $\tau^{+}$mutually almost disjoint subsets; i.e., a family $\left\{S_{\alpha} \mid \alpha<\tau^{+}\right\}$ so that for $a<\beta<r^{+}$

$$
\left|S_{\alpha} \cap S_{\beta}\right|<\tau
$$

If $\kappa$ is subtle, $\kappa$ is actually strongly inaccessible: For if $\lambda$ is a cardinal so that $\lambda<\kappa \leq 2^{\lambda}$, apply the definition of subtlety to the sequence $\left(s_{a} \mid \alpha<\kappa\right)$ where the $S_{a}$ for $a \geq \lambda$ are distinct subsets of $\lambda$. However, we shall show that certain large cardinal assumptions imply the existence of nonstrongly inaccessible ethereal cardinals.

A final comment on our definitions: If $\kappa$ is regular, $\diamond(\kappa)$ holds if and only if there is a family $\left\{T_{x} \mid X \subseteq \kappa\right\}$ of Mahlo subsets of $\kappa$ so that for any $\alpha, X, X^{\prime} \subseteq \kappa$

$$
\alpha \in T_{\boldsymbol{x}} \cap T_{\boldsymbol{x},} \rightarrow X \cap a=X^{\prime} \cap a .
$$

For if $(*)$ holds, we can define the $S_{a}$ in a unique fashion by

$$
S_{a}=X \cap a \text { for some } X \text { s.t. } a \in T_{x}
$$

and if the $\left(S_{\alpha} \mid \alpha<\kappa\right)$ are given, define $T_{X}$ by

$$
T_{X}=\left\{\alpha \mid X \cap \alpha=S_{\alpha}\right\} .
$$

Hence, if $\diamond(\kappa)$ holds, $\left\{T_{x} \mid x \subseteq \kappa\right\}$ is a family of almost disjoint Mahlo sets of power $.2^{\kappa}$ and the closed unbounded filter on $\kappa$ is not $\lambda$-saturated for any $\lambda \leq 2^{\kappa}$.

2. Ethereal cardinals. The following result gives a useful reformulation of Definition 1.2:

2.1. Proposition. If $\alpha$ is regular, the following statements are equivalent:

(1) $\kappa$ is ethereal.

(2) For any closed unbounded $C \subseteq \kappa$, sequence $\left(S_{\alpha} \mid \alpha<\kappa\right)$ so that $S_{\alpha} \subseteq \alpha \times \alpha$, $\left|S_{a}\right|=|a|$, there exist $a<\beta, a, \beta \in C$ so that

$$
\left|s_{\alpha} \cap s_{\beta}\right|=|\alpha| \text {. }
$$

(3) For any closed unbounded $C \subseteq \kappa$, sequences $\left(S_{\alpha} \mid \alpha<\kappa\right),\left(C_{a} \mid \alpha<\kappa\right)$ so that $S_{a} \subseteq \alpha,\left|C_{a}\right|=\left|S_{a}\right|=|\alpha|, C_{a}$ closed unbounded in a there exist $\alpha, \beta \in C$ so that 


$$
a \in C_{\beta} \text { and }\left|S_{a} \cap S_{\beta}\right|=|a| \text {. }
$$

As we shall see later, we can require $\alpha, \beta$ to be regular cardinals $<\kappa$ in both (2) and (3).

Proof. Obviously, (2) and (3) imply (1). To show that (1) implies (2), let $\phi: \kappa \times \kappa \rightarrow \kappa$ be a one-to-one function. Then the set

$$
D=\left\{\alpha \mid \phi^{\prime \prime}(\alpha \times \alpha) \subseteq \alpha\right\}
$$

is closed unbounded. Given $C \subseteq \kappa, S_{\alpha} \subseteq a \times a$ we can then apply (1) to $C \cap D$ and $\phi^{\prime \prime}\left(S_{a}\right)$.

To deduce (3) from (1), let $C \subseteq \kappa, S_{a}, C_{a} \subseteq \alpha(a<\kappa)$ be as in (3), and $f_{a}$ : $a \rightarrow \alpha$ enumerate $S_{\alpha}$. Let

$$
t_{a}=\left\{\left(\delta, f_{a}(\delta)\right) \mid \delta \in C_{a}\right\}
$$

Then $t_{\alpha} \subseteq \alpha \times a$. We can apply (2) to the sequence $\left(t_{\alpha} \mid a<\kappa\right)$ with $\{\alpha \mid a \in C$ a cardinal\} as the closed unbounded set.

We shall now investigate the 'size' of ethereal cardinals. The following definition proves to be instrumental in this endeavor:

2.2. Definition. Let $\kappa$ be ethereal. A set $X \subseteq \kappa$ is big if for every sequence $\left(S_{a} \mid a<\kappa\right)$ so that $S_{a} \subseteq a,\left|S_{a}\right|=|a|$ closed unbounded $C \subset \kappa$, there exist $\alpha$, $\beta \in C$ so that $a<\beta$ and

$$
a \in X \text { and }\left|S_{\alpha} \cap S_{\beta}\right|=|a| \text {. }
$$

2.3. Proposition. Let $\kappa$ be ethereal.

(1) If $X \subseteq K$ is big, for every sequence $\left(S_{\alpha} \mid \alpha \subset \kappa\right)\left(S_{\alpha} \subseteq \alpha\right)$, closed unbounded $C$ there exist $\alpha, \beta \in C \cap X$ so that $\alpha<\beta$ and $\left|S_{\alpha} \cap S_{\beta}\right|=|\alpha|$.

(2) If $Y$ is big and $X \subseteq Y$ is not big, $Y-X$ is big.

(3) If $X$ is big, $f: X \rightarrow \kappa$ a pressing down function, then there is a $\xi<\kappa$ so that $f^{-1}(\{\xi\}) \cap X$ is big.

Proof. (1) Given the $S_{\alpha}$ 's, apply Proposition 2.1(2) to

$$
t_{a}= \begin{cases}\{0\} \times S_{a} & \text { if } a \in X, \\ \{1\} \times S_{a} & \text { if } a \notin X .\end{cases}
$$

(2) This obviously follows from (3).

(3) Suppose that for no $\xi<\kappa f^{-1}(\{\xi\}) \cap X$ is big. Let $C_{\xi}$, $\left(S_{a}^{\xi} \mid a<k\right)$ be the offending sets in question. Let $E$ be the diagonal intersection of $C_{\xi}$ 's; i.e., let

$$
E=\left\{a \mid \forall \xi: \xi<a \rightarrow a \in C_{\xi}\right\}
$$


For $a \in f^{-1}(\{\xi\}) \cap X$, let

$$
S_{a}=\{\xi\} \times S_{a}^{\xi}
$$

Then, if $\alpha, \beta \in E$ so that $\alpha<\beta$ and $\left|S_{a} \cap S_{\beta}\right|=|\alpha|$, we must have $f(\alpha)=$ $f(\beta) \stackrel{\text { def }}{=} \xi$. But then $\alpha, \beta \in C_{\xi}$ : a contradiction.

- For an ethereal cardinal $\kappa$, let $F_{\kappa}$ be the filter of all subsets of $\kappa$ which are complements of nonbig sets. Thus, by the above, $F_{K}$ is a normal, $\kappa$-complete filter. The following proposition will show in particular that $F_{K}$ is not the closed unbounded filter.

2.4. Theorem. If $\kappa$ is ethereal, then

(1) $\kappa-M_{0}$ is not big.

(2) If $A$ is big, then $M(A)$ is big, where

$$
\mathcal{M}(A) \stackrel{\text { def }}{=}\{a \mid A \cap a \text { is Mablo }\} \text {. }
$$

Moreover, $A-M(A)$ is not big.

(3) $\kappa$ is in the $\kappa$ tb Mablo class.

Here we define the Mahlo classes $M_{a}$ by induction as follows:

$$
M_{0}=\{\gamma \mid \gamma \text { regular }\}, \quad M_{a_{+1}}=M\left(M_{\alpha}\right), \quad M_{\lambda}=\bigcap\left\{M_{a} \mid \alpha<\lambda\right\} \quad(\lambda \text { limit }) .
$$

Proof of theorem. (1) Suppose that the set $S=\{a \mid a<\kappa$ singular cardinal $\}$ is big. By Proposition 2.3(3), there is a regular $\xi<\kappa$ so that the set

$$
X=\{\alpha<\kappa \mid \operatorname{cof}(\alpha)=\xi \text { and } \alpha>\xi\}
$$

is big. For $a \in X$, let $S_{a}$ be a cofinal sequence of type $\xi$ in $a$, and let

$$
t_{a}=\left\{(\beta, \eta) \mid \beta \in S_{a} \text { and } \eta<\beta<\alpha\right\} .
$$

By Proposition 2.3(1) we can then find $a, \beta \in X$ so that $a<\beta$ and $\left|t_{\alpha} \cap t_{\beta}\right|=$ $|a|$. Hence, $S_{\alpha} \cap S_{\beta}$ must be cofinal in $\alpha$. But this means that $S_{\beta} \cap \alpha$ is of type $\xi$; a contradiction.

(2) Assume that $A-M(A)$ is big. By (1) we can without a loss of generality assume that $A \subseteq M_{0}$. For any $a \in A-M(A)$, let $C_{a} \subseteq \alpha$ be closed unbounded so that $C_{\alpha} \cap A=0$. Since $A-M(A)$ is big, we can find $\alpha<\beta, \alpha, \beta \in A-M(A)$, so that $C_{\alpha} \cap A=C_{\beta} \cap A=0$ and $\alpha \in C_{\beta}$ by Proposition 2.3 (1). But then $\alpha \in C_{\beta} \cap A$ : a contradiction.

(3) This follows trivially from (1) and (2).

Open question. Can $F_{K}$ ever be $k$-saturated?

This obviously cannot be the case if, for example, we have $\kappa$ distinct normal ultrafilters on $\kappa$.

As a corollary, we obtain 
2.5. Proposition. If $\kappa$ is regular, the following statements are equivalent:

(1) $\kappa$ is etbereal.

(2) For every closed unbounded $C \subseteq \kappa$ sequence $\left(S_{\alpha} \mid \alpha<\kappa\right)$ s.t. $S_{\alpha} \subseteq \alpha$ bas cardinality $|\alpha|$, there exist $\alpha, \beta$ regular so that $\alpha<\beta$ and $\alpha, \beta \in C$ and $\left|s_{a} \cap s_{\beta}\right|=\alpha$.

In case of strong inaccessibility, being ethereal coincides with subtlety:

2.6. Proposition. If $k$ is strongly inaccessible, $\alpha$ is ethereal if and only if $\kappa$ is subtle. In particular, if $\kappa$ is subtle and $X \subseteq \kappa$ a big set, for any closed unbounded $C \subseteq \kappa$, sets $S_{\alpha} \subseteq \alpha(\alpha<\kappa)$ there exist $\alpha<\beta$ so that $\alpha, \beta \in C \cap X$ and $S_{a}=S_{\beta} \cap a$.

Proof. For suppose that $\kappa$ is ethereal and $X \subseteq \kappa$ is big. Let

$$
\psi: \bigcup\{P(\alpha) \mid a<\kappa\} \rightarrow \kappa
$$

(here $P(\alpha)$ denotes the power-set of $\alpha$ ) be a one-to-one function, and

$$
f(\delta) \stackrel{\text { def }}{=} \sup \{\psi(A) \mid A \subseteq \delta\} \quad(\delta<\kappa) .
$$

Given the sets $C, S_{a} \subseteq \alpha$, let

$$
D=\{a<\kappa \mid a \in C \text { a cardinal and } \forall \delta: \delta<a \rightarrow f(\delta)<a\} .
$$

For $a \in D$, let

$$
t_{a}=\left\{\psi\left(S_{a} \cap \delta\right) \mid \delta<a\right\}
$$

Apply Proposition 2.5(2) to the sets $C,\left(t_{a} \mid a<\kappa\right)$.

R. Jensen and $K$. Kunen have independently shown that if $\kappa$ is subtle, $\diamond(\kappa)$ holds. We shall generalize this remark to ethereal cardinals. There is, however, an obvious restriction: If $\diamond(\kappa)$ holds, then it is easy to see that $2^{\kappa}=\kappa$. Here $2^{K}$ denotes the weak power of $\kappa$ :

$$
2^{\kappa}=\bigcup\left\{2^{a} \mid a<\kappa\right\} .
$$

The above property may fail for some ethereal cardinals. However, we can prove a weaker property, slightly reminiscent of property $(*)$ of $\$ 1$.

2.7. Proposition. If $k$ is ethereal, then there exist sets $S_{\alpha} \subseteq \alpha(\alpha<\kappa)$ so that for every $X \subseteq \kappa$ of cardinality $\kappa$ the set

$$
P_{X}=\left\{a|| X \cap S_{a} \mid=\alpha, \text { a regular cardinal }\right\}
$$

is Mablo.

Proof. Define sets $C_{a}, S_{\alpha}$ by induction as follows: Given $S_{\beta}(\beta<\alpha)$, if there is a set $S \subseteq \alpha$ of cardinality $\alpha$ and a closed unbounded $C \subseteq \alpha$ so that for any $\beta$, 


$$
\beta \in C \rightarrow\left|S \cap S_{\beta}\right|<|\beta|,
$$

then set $S_{a}=S, C_{a}=C$ for some such pair $\langle S, C\rangle$. Otherwise, set $S_{a}=C_{a}=a$.

Given $X \subseteq \kappa$, if the corresponding set $P_{X}$ is not Mahlo, let $C$ be closed unbounded so that

$$
a \in C \rightarrow\left|X \cap S_{a}\right|<|a|, \quad|X \cap a|=|a| .
$$

Then for every $\alpha \in C$, a pair $\langle S, C\rangle$ as described above exists. Thus, for $\beta<\alpha$, $\beta, \alpha \in C \cap M_{0}$

$$
\beta \in C_{\alpha} \rightarrow\left|S_{\beta} \cap S_{a}\right|<\beta
$$

But this contradicts Proposition 2.1(3).

2.8. Theorem. If $\alpha$ is ethereal and $2^{\kappa}=\kappa$, then $\diamond(\kappa)$ bolds.

Actually, the method of proof will show that if $\kappa$ is ethereal and there is a family $\left\{\left.X_{\gamma}\right|_{\gamma}<\kappa\right\}$ of almost disjoint sets so that for every $a<\kappa$ the set $S_{a}=$ $\left\{X_{y} \cap a \mid \gamma<\kappa^{+}\right\}$has cardinality $\leq \kappa$, then there is a family of $\kappa^{+}$almost disjoint Mahlo sets.

Proof of Theorem 2.8. Pick one-to-one functions $\phi_{a}: P(\alpha) \rightarrow \kappa(\alpha<\kappa)$ so that the sets $X_{a}=\phi_{a}^{\prime \prime}(P(\alpha))(\alpha<\kappa)$ are pairwise disjoint and $X_{a} \subseteq(\alpha, k)$. Define a function $p$ on the set $X=\bigcup\left\{X_{a} \mid \alpha<\kappa\right\}$ by setting $p=a$ on $X_{a}$. Thus, $p<$ id on $X$. Given any set $S \subseteq \kappa$, the set

$$
Q_{s}=\left\{\phi_{\alpha}(S \cap \alpha) \mid \alpha<\kappa\right\}
$$

has cardinality $\kappa$ and

Also, for any $\gamma<\kappa, S, S^{\prime} \subseteq \kappa$

$$
p: Q_{S} \underset{\text { onto }}{\stackrel{1-1}{\longrightarrow}} \kappa
$$

$$
\gamma \in Q_{S} \cap Q_{S^{\prime}} \rightarrow S \cap p(\gamma)=S^{\prime} \cap p(\gamma) .
$$

Let us call a set $S \subseteq K$ a $\phi$-set if for some $t \subseteq a \leq \kappa$

$$
S=\left\{\phi_{\gamma}(t \cap \gamma) \mid \gamma<a\right\} \text {. }
$$

Define sets $\bar{S}_{\alpha} \subseteq \alpha$ inductively: Given the $\bar{S}_{\beta}$ for $\beta<\alpha$, if there exists a $\phi$-set $S \subseteq a$ so that $\left|S \cap \bar{S}_{\beta}\right|<|\beta|$ on a closed unbounded set of $\beta$ 's in $\alpha$, let $\bar{S}_{\alpha}=$ such an $S$. Otherwise, let $\bar{S}_{\alpha}=a$. For $X \subseteq \kappa$, let

$$
N_{X}=\left\{a|| Q_{X} \cap \bar{S}_{a} \mid=\alpha, \bar{S}_{a} \text { a } \phi \text {-set, } a \text { regular }\right\} .
$$

Then, for each $X \subseteq \kappa$ the set $\left\{a \mid Q_{X} \cap \alpha\right.$ contains a $\phi$-set of cardinality $\left.a\right\}$ is closed unbounded and therefore the set $N_{X}$ is Mahlo. 
Now if $a \in N_{X} \cap N_{X^{\prime}}$ for some $X, X^{\prime} \subseteq \kappa$, both $Q_{X} \cap \bar{S}_{a}$ and $Q_{X^{\prime}} \cap \bar{S}_{a}$ have cardinality $a$. Since $\bar{S}_{a}$ is a $\phi$-set, for some $\xi \geq a, t \subseteq \xi$ :

$$
\bar{s}_{a}=\left\{\phi_{\gamma}(t \cap \gamma) \mid \gamma<\xi\right\} \text {. }
$$

Thus, both sets $\{y \mid X \cap y=t \cap y\},\left\{\gamma \mid X^{\prime} \cap y=t \cap \gamma\right\}$ have cardinality $\geq a$. Since these sets are initial segments, $X \cap a=X^{\prime} \cap a$. We have established: For any $a<\kappa$,

$$
a \in N_{X} \cap N_{X^{\prime}} \rightarrow X \cap a=X^{\prime} \cap a
$$

$\diamond(\kappa)$ then follows by the remarks of $\$ 1$.

The following result (involving a large cardinal property) yields a wide variety of nonstrongly inaccessible ethereal cardinals. It is clearly analogous to the fact that every measurable cardinal is subtle.

2.9. Theorem. If $\kappa$ carries a $\lambda$-saturated $\kappa$-complete filter for some $\lambda<\kappa$, then $\kappa$ is ethereal. In particular, if $2^{\mathcal{K}}=\kappa, \diamond(\kappa)$ bolds.

Proof. For if $\kappa$ satisfies the hypothesis of the theorem, by Solovay [3], we can find a normal $\lambda$-saturated $\kappa$-complete filter $\pi$ over $\kappa$ Then

$$
P=\{a \mid a \text { regular cardinal }\}-\lambda \epsilon \pi \text {. }
$$

If $\kappa$ is not ethereal, let $C,\left(S_{a} \mid a \in P\right)$ be the offending sequences in question. Let

$$
X_{\delta, \alpha}=\left\{\beta \mid \beta \in P \text { and } \alpha<\beta \rightarrow \operatorname{Sup}\left(S_{\alpha} \cap S_{\beta}\right)<\delta\right\} \quad(\delta<\alpha<\kappa) .
$$

For $a \in P \cap C$, we can find an $f(\alpha)<a$ so that $X_{f(a), a} \in \boldsymbol{r}$. Thus, there is a $\xi<\kappa$ and a $Y \in \gamma$ so that $Y \subseteq P \cap C$ and $f \leq \xi$ on $Y$. Let

$$
Y_{0}=\left\{\alpha \in Y \mid \beta<\alpha \text { and } \beta \in Y \rightarrow \alpha \in X_{\xi, \beta}\right\}
$$

Then, by normality of $r, Y_{0} \in \Re$ and for $\alpha, \beta \in Y_{0}$

$$
\alpha<\beta \rightarrow \operatorname{Sup}\left(S_{\alpha} \cap S_{\beta}\right) \leq \xi
$$

a contradiction, since $Y_{0} \in \pi$ and by another normality argument we can find an $\eta<\xi$ so that $\eta \in S_{\alpha} \cap S_{\beta}$ for some $a<\beta, \alpha, \beta \in Y$.

3. The closed unbounded filter. It is a well-known open question whether the closed unbounded filter on a regular cardinal $\kappa$ can ever be $\kappa^{+}$-saturated. Kenneth Kunen [2] has shown that if for example the closed unbounded filter on $\omega_{1}$ is $\omega_{2}$-saturated, then Solovay's $0^{+}$exists. The following observation throws out some other cases. 
3.1. Theorem. If $2^{\kappa}>\kappa^{+}$and $\kappa$ is regular and the set $M=\left\{\left.a\left|2^{|a|}=\right| a\right|^{+}\right\}$is Mablo in $\kappa$, then there is a family of $\kappa^{+}$almost disjoint Mablo sets in $\kappa_{0}$

In particular, if $2^{\omega_{1}}>\omega_{2}$ and $2^{\omega}=\omega_{1}$, the closed unbounded filter on $\omega_{1}$ is not $\omega_{2}$-saturated. The same conclusion can also be made if $\omega_{1}<2^{\omega}<2^{\omega_{1}}<\omega_{\omega}$. (This follows from a simple modification of the proof of Theorem 3.1.)

Proof of Theorem 3.1. Let $D$ be an ultrafilter extending the closed unbounded filter s.t. $D \ni M$. If $a \in M$, let

$$
P(\alpha)=\left\{\left.S_{a}^{\nu}|\nu<| \alpha\right|^{+}\right\} .
$$

For any $S \subseteq \kappa$, define $f_{S}: M \rightarrow \kappa$ by

$$
f_{s}(a)=\text { least } \nu \text { s.t. } s \cap a=s_{\alpha}^{\nu} \text {. }
$$

Thus, $P=\left\{f_{S} \mid S \subseteq \kappa\right\}$ is a family of $2^{k}$ eventually different functions. Since $P$ is linearly ordered in the ultrapower given by $D$, there is a function $f: k \rightarrow \kappa$ so that, for every $a<\kappa, f(a)<|a|^{+}$and the set

has cardinality $\geq \kappa^{+}$. Let

$$
P^{*}=\left\{S \mid f_{S} \leq f(\bmod D)\right\}
$$

$$
\left\{t_{a}^{\nu} \mid 0<\nu<a\right\}=\left\{S_{a}^{\nu} \mid \nu<f(a)\right\} .
$$

For $S \in P^{*}$, define a pressing down function $t_{S}$ by

$$
t_{s}(\alpha)=\left\{\begin{array}{l}
\text { least } \nu<a \text { so that } S \cap a=t_{\alpha}^{\nu} \text { if such a } \nu \text { exists, } \\
0 \text { otherwise. }
\end{array}\right.
$$

For $S \in P^{*}$, pick a Mahlo set $M_{S}$ and an ordinal $\gamma_{S}>0$ so that $t_{S}=\gamma_{S}$ on $M_{S}$. Let $P^{* *} \subseteq P^{*}$ be a family of power $\kappa^{+}$and $\gamma>0$ so that, for each $S \in F^{* *}, \gamma_{S}=\gamma$. Then $\left\{M_{S} \mid S \in P^{* *}\right\}$ is the desired family of Mahlo sets.

\section{REFERENCES}

1. R. Jensen, Some conbinatorial properties of $L$ and $V$ (to appear).

2. K. Kunen, Some applications of iterated ultrapowers in set-theory, Ann. Math: Logic 1 (1970), 179-227. MR 43 \#3080.

3. R. Solovay, Real-valued measurable cardinals, Proc. Sympos. Pure Math., vol. 13, Part 1, Amer. Math. Soc., Providence, R. I., 1971, pp. 397-428. MR 45 \#55.

DEPARTMENT OF MATHEMATICS, UNIVERSITY OF CALIFORNIA, BERKELEY, CALIFORNIA 94720 